\title{
Operational Analysis in the Launch Environment
}

\author{
George James, Mo Kaouk, Tim Cao, Vince Fogt, Rodney Rocha, \\ Ken Schultz, Jon-Michael Tucker, and Eli Rayos \\ Loads and Structural Dynamics Branch \\ NASA Johnson Space Center \\ Houston, Texas 77058 \\ Jeff Bell \\ ESCG Jacobs \\ Houston, Texas 77058 \\ David Alldredge and Tom Howsman \\ Dynamic Concepts Incorporated \\ Huntsville, Alabama 35806
}

\begin{abstract}
The launch environment is a challenging regime to work due to changing system dynamics, changing environmental loading, joint compression loads that cannot be easily applied on the ground, and control effects. Operational testing is one of the few feasible approaches to capture system level dynamics since ground testing cannot reproduce all of these conditions easily. However, the most successful applications of Operational Modal Testing involve systems with good stationarity and long data acquisition times.

This paper covers an ongoing effort to understand the launch environment and the utility of current operational modal tools. This work is expected to produce a collection of operational tools that can be applied to non-stationary launch environment, experience dealing with launch data, and an expanding database of flight parameters such as damping. This paper reports on recent efforts to build a software framework for the data processing utilizing existing and specialty tools; understand the limits of current tools; assess a wider variety of current tools; and expand the experience with additional datasets as well as to begin to address issues raised in earlier launch analysis studies.
\end{abstract}

\section{INTRODUCTION}

The spacecraft launch environment is a highly complex and non-stationary event that is characterized by high amplitude input forces, highly variable loads, a wide spectrum of responses, constantly changing vehicle mass, active control interactions, staging, and limited instrumentation. At the same time, structural response analyses and loads estimations must be performed with models that are only partially validated using ground test data due to the fact that access to diagnostic and environmental ground tests are limited. To compound matters, projects are tending to use reduced uncertainty factors designed to protect for loads increases and model unknowns. As a result, the designs progress rapidly before loads and structural problems are uncovered. This means that there are very few tools available to recover from structural dynamics issues in such a dynamic environment without costly redesigns late in the design cycle or in early operations.

The inclusion of operational modal testing and analysis tools can be used to offset these limitations by providing more cost and schedule effective opportunities for diagnostic information extraction. These opportunities are available on the ground and during flight as well as on full-up systems, subsystems, and components. Since the ability to obtain good modal information is limited by ground test availability, the flight data from early test flights and early operational flights must be used to estimate such values. Also, there are many more additional opportunities for modal information during manufacturing, environmental test, transportation, and natural excitation events.

A hypothetical vehicle development program could be envisioned to use Operational Modal Analysis (OMA) in conjunction with traditional Experimental Modal Analysis (EMA) from the onset. It is likely that a technically advantageous level of diagnostic testing could then be injected into the program. EMA would be heavily used in the early stages of the program when a multitude of small-scale development tests could easily and inexpensively be injected into the schedule and operational testing opportunities are limited. As the program matures, it becomes more difficult (but no less impor- 
tant) to inject diagnostic testing into the work flow but there are increasing opportunities to obtain operational data. In the final stages of flight and operations, it becomes virtually impossible to exercise traditional test options and operational testing becomes the primary tool. Figure 1 illustrates this notional relationship between EMA and OMA techniques.

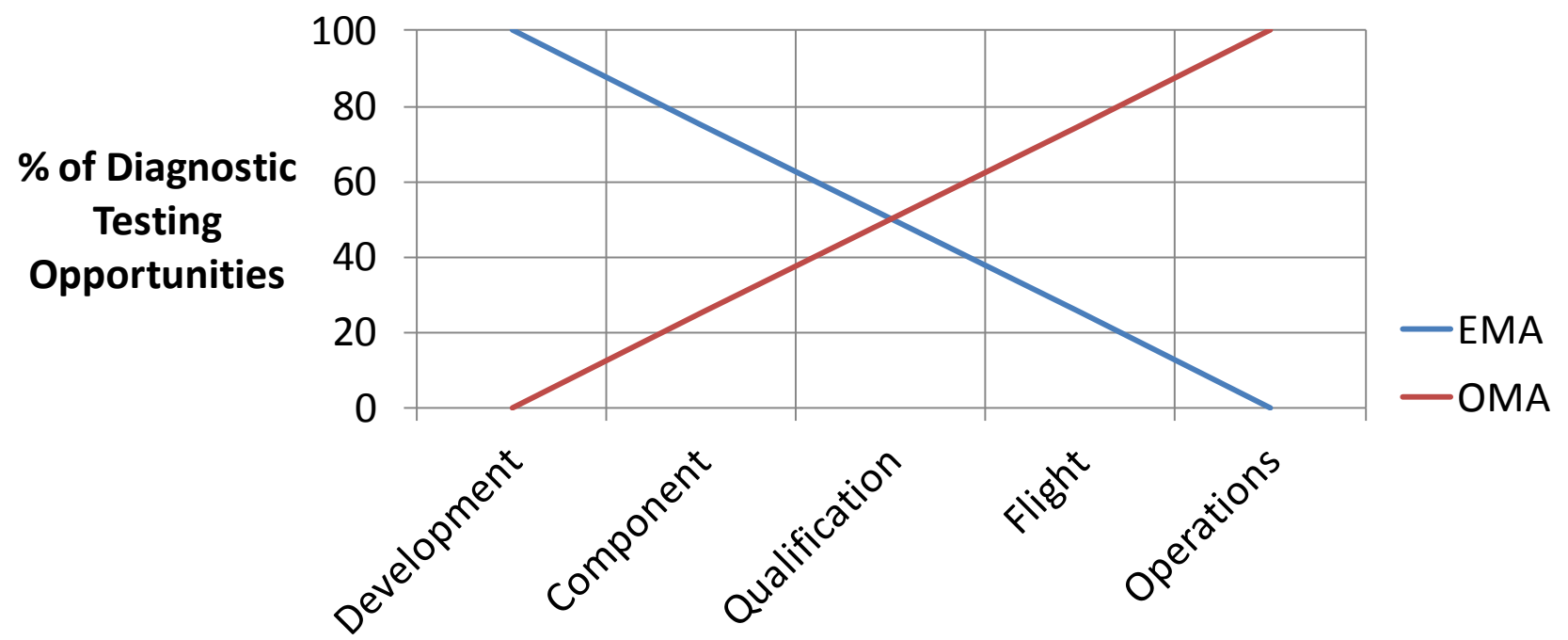

Program Milestones

Figure 1. Notional Relationship between EMA and OMA over the Life of a Hypothetical Program

The reality of the OMA state of the art is such that not all of the available opportunities are at the same level of technical readiness. Most of the OMA testing opportunities over the life of a program will be traditional well-behaved situations where long dwell times with a steady system can drive standard OMA tools. These situations include such opportunities as environmental vibration testing, transportation-induced loading, or wind-excited pad stays. The data from these opportunities can be consistently processed with current tools and the technology is well in hand and validated.

Alternatively, there are a few of situations where OMA opportunities involve situations where the tools are available for use but validation activities still need to be performed as the data has some inherent difficulties. Examples of these types of situations involve full scale flight data where the launch system may be unsteady (due to fuel mass loss) and the environment is uncertain but the loading is very impulsive in nature such as at pad release, staging, engine cut-off, or orbital thermal transitions. The data produced in these situations will be available in short time segments and will include some inherent noise sources. However, the impulsive nature of the loading will not tax the operational algorithms as the data will appear to be very traditional in nature. Some bench-top mechanism or non-linear isolator tests would also fall into this category of OMA opportunities with well-developed tools in-hand but in need of validation activities.

And finally there are some situations where OMA tool development is required. The bulk of the vehicle launch phase is one such opportunity where the unsteady system properties coupled with random loading are taxing the technical readiness. The pressure to process short time records with little averaging to extract modal damping is a major issue to be overcome. Most of this paper will be devoted to this group's efforts to address these launch issues. Another issue of similar need for development involves those opportunities where data takes from high-frequency acoustics tests need to be processed to extract structural dynamics parameters for vibroacoustics codes. The ability to extract parameters (such as modal density, damping loss factors, coupling loss factors, input power) from high frequency/high modal density data is an area of active research. 


\section{TECHNICAL BACKGROUND}

The activities underway to inject OMA into future spacecraft programs by the authors of this work include the following:

1. Develop a code to provide basic data analysis functions and a framework for multiple OMA modules;

2. Module development of multiple OMA algorithms;

3. Processing of a wide-range of available datasets with OMA tools to develop operational experience;

4. Use of analytical datasets (simple numerical and full launch vehicle models) to understand specific issues; and

5. Perform controlled analyses as needed to validate that the OMA tools are operationally ready for use.

\section{Code Development}

There are multiple precursor steps needed in the analysis of an OMA data set that are common to multiple approaches and even to traditional EMA. These steps include reading in and converting the data, viewing the data, performing statistical and data quality assessments, breaking up the data into increments for analysis and/or averaging, converting to and from the frequency domain, calling analysis modules, resynthesizing the original data, etc. It is critically important to have such a common framework in place to implement a wide range of unique tools (OMA in this case) at many entry points in a program development activity by a team of semi-independent engineers. The acronym chosen for this software framework is MIDOS (Modal Identification of Dynamic Operational Systems) and is coded in MATLAB. MIDOS is currently functional for signal processing and data quality reviews and limited modal analysis capability.

\section{Module Development}

The multitude of entry points for OMA in a program development; the different needs for the multitude of anticipated programs; the multitude of different responsible engineers; and the need for different OMA techniques to help offset the inherent uncertainties in the technology all drive the requirement to have an open framework that allows the implementation of a multitude of OMA tools. Hence a major activity involved the development of individual modules to implement a single technique for integration into the MIDOS framework. The techniques currently developed, in development, or under consideration include: correlation function processing [1,2], Stochastic Subspace Identification (SSI) [3], Frequency Domain Decomposition (FDD) [4], wavelet processing [5], Hilbert-Huang [6], force reconstruction [7,8] as well as other supporting techniques such as mode shape extraction [9], harmonic removal [10], Maximum Entropy Method (MEM) [11,12], random decrement [13,14], etc. Currently, there are limited modules built for standard OMA processing as well as unsteady launch processing based on correlation functions. Modules based on other more advanced approaches are still in development.

\section{Data Processing}

A feature of this effort is the early emphasis on building some operational experience with real data sets to drive the subsequent code and module development activities. It is expected that this data processing emphasis will continue throughout the life of this project when new and updated modules are developed, integrated into MIDOS, and subjected to validation exercises; when new datasets are made available; and when new engineers have a need or desire to exercise the tools. The primary datasets used to exercise these modules or code includes the following:

1. Space Shuttle flight [15],

2. Space Shuttle rollout [8],

3. International Space Station (ISS),

4. RR-1 lander tethered test flight [1],

5. PA-1 test flight [1],

6. Ares 1-X roll-out,

7. Ares 1-X flight [16],

8. Ares 1-X Liftoff,

9. Acoustic panel tests,

10. Orion Ground Test Article (GTA) acoustics Environmental Correlation Test (ECT), and

11. Simplified analytical models [1].

As of the writing of this paper, several other data sets are being considered for inclusion in this list including (but not limited to) aircraft, launch vehicle, and ground vibration test sources. The analysis activities related to datasets \#8 and \#11 above will be discussed in more detail in this paper. Special emphasis will be paid to lessons-learned by previous 
insights driven by analysis activities related to datasets \#4,\#5, \#6, and \#7. A question of particular interest to this paper are the indications from the previous database processing exercises that the control system is affecting the estimated modal properties of the lowest bending modes during launch [1].

\section{Analytical Studies}

Another aspect of this development activity to date, has been the use of extremely simplified analytical models (one or two Degrees Of Freedom or DOF) to study specific questions - usually posed by the dataset processing activities. Reference [1] covers some of these activities. A particularly vexing problem in launch analyses that have been studied with simplified analytical models is the "beating" phenomena (unexplained but repeated increases in correlation) seen in the correlation-based analysis approaches. The need for more complex analytical data sets has become apparent with the need to study control system interaction effects. Hence the use of analytical datasets with full-up launch vehicle models and liftoff/ascent tools has recently been added to the list of ongoing studies.

\section{Validation Activities}

The validation of the available tools has not begun in earnest as of this writing. However, the datasets are being collected that will eventually be used to enable the needed validation. Formal validation will take place over time but should be subjected to a complete documentation to allow for a true reference for these techniques. The expected validation activities will involve some or all of the following:

1. Comparison of MIDOS/OMA results with traditional EMA results for simple systems;

2. Comparison of MIDOS/OMA results with known analytical solutions for simple and complex models in the launch environment;

3. Comparison of MIDOS/OMA results from different MIDOS/OMA techniques on the same data set; and

4. Comparison of MIDOS/OMA results with results from independent analysts and implementations.

The current plan is to develop a full plan for validation after MIDOS is nearer to completion.

\section{Status of Launch Environment Processing}

There are two primary issues requiring near term attention that have resulted from the previous studies involving the analysis of launch data [1]: (1) understanding the interaction with the control system; and (2) understanding and solving the short time record "beating" phenomenon. The remainder of the paper will focus on the recent work on these issues.

\section{BEATING PHENOMENA}

The most significant complexity associated with operational analysis of launch systems is the unsteadiness due to rapidly changing mass properties. This usually drives the available time records to be very short due to the need to utilize some type of sliding window analysis. The analysis of several recent data sets (RR-1, PA-1, Ares1-X, simple analytical) has shown that one effect (when using the correlation-based processing approaches) is a beating-like phenomena which limits the amount of the correlation functions that can be used for processing [1,16]. Although the first few low-lag points in the correlation functions are relatively unaffected, the higher-lag time data points can be relatively useless for analysis. Figure 2 illustrates the beating phenomena as illustrated by a simple one DOF analytical model of a $10 \mathrm{~Hz}$ mode excited by random white noise. The random input is shown in the top plot of Figure 2. The displacement response of the $10 \mathrm{~Hz}$ system is shown in the middle plot. Displacement is used as opposed to the more easily measured acceleration as it illustrates the issue with more clarity. Notice that the response shows random excitations of the $10 \mathrm{~Hz}$ system mode, which eventually damps out. The lower plot provides the autocorrelation function of the displacement shown in the middle plot. The beating phenomena are clearly seen as the correlation increases at longer lags. Note that the "beating" terminology is adopted as the correlation function looks like a time history of closely spaced modes interacting or "beating". The repetitive increases in correlation deviates from the theoretical damped sinusoids (as expected from OMA/NExT correlation functions [17]) and limit the ability to separate closely-spaced modes as only early-lags can be analyzed. 

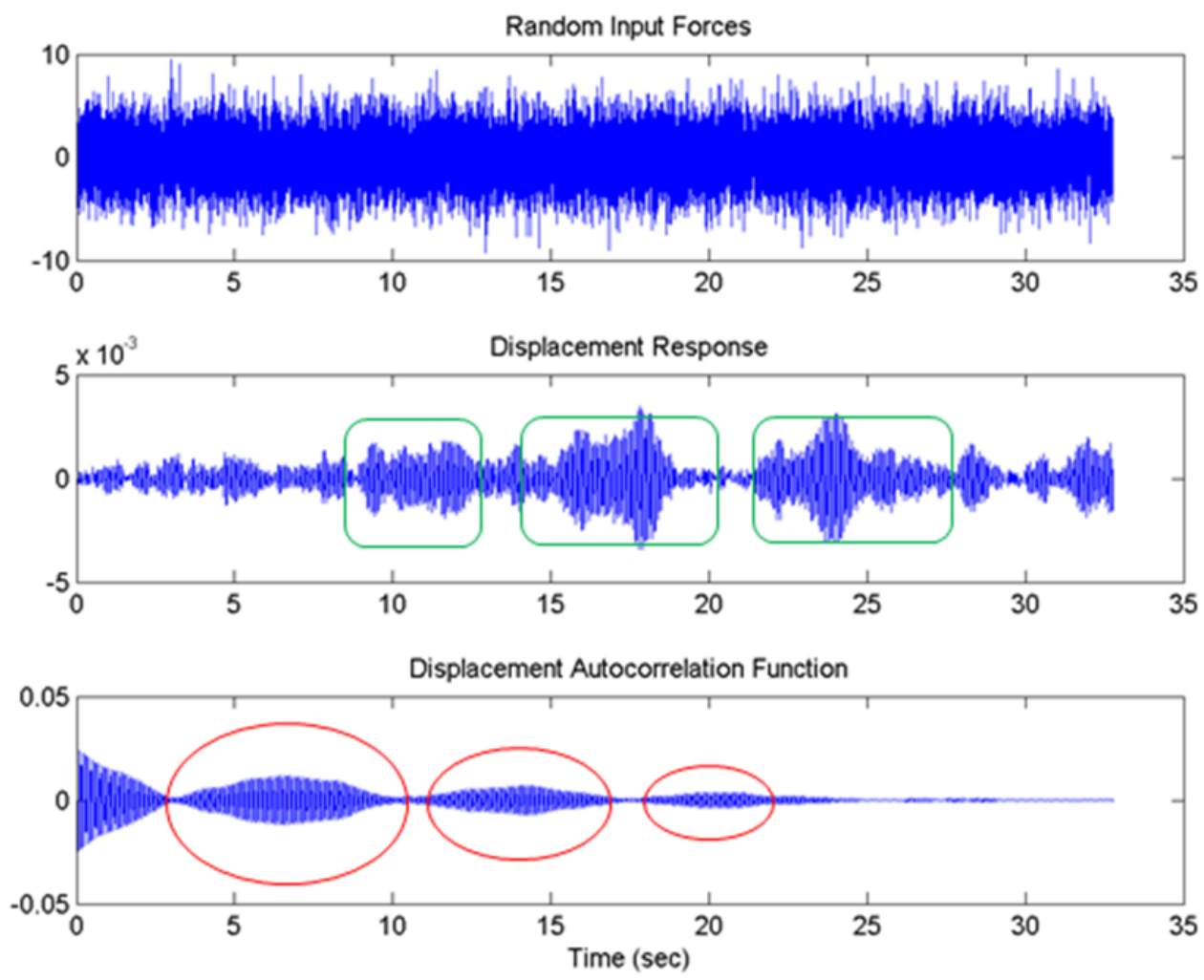

Figure 2. Beating Phenomena in Correlation Functions as Illustrated with Single DOF Analytical Data

As of the production of Reference [1], the authors did not have a working hypothesis on the cause of this beating phenomenon. Also, a trial solution to the issue was attempted by summing the different cross and auto-correlation functions in an attempt to reduce the "beats". After performing additional analytical studies, a working hypothesis is now available. The beating seen in the correlation functions are a numerical increase in correlation due to the random increases in modal responses due to the input excitation. Given this hypothesis, the idea of summing different correlation functions from different sensors is not expected to significantly reduce the beating effects as the random modal excitations would be global in nature and appear in most of the correlation functions.

The most obvious approach to reduce the beating phenomena is to generate correlation functions with a technique that allows frequency domain averaging or by averaging correlation functions in the time lag domain generated at different times [1]. Figure 3 shows the effects of this averaging approach. This approach is completely viable for traditional operational modal situations as time record lengths are sufficient to allow multiple averages (either in the time lag domain or the frequency domain). However, for the launch analyses, this approach is not viable due to the limited time records.

An alternative approach to reduce the beating effects is via repeated correlation calculations using the same parent time data. Figure 4 shows the effectiveness of this approach. The top plot shows an autocorrelation function of a $10 \mathrm{~Hz}$ single DOF system excited randomly. If a another correlation calculation is performed using the first autocorrelation function as the parent data then the correlation function shown in the middle plot results. The bottom plot results after performing an additional eight correlation calculations using the function shown in the middle plot as the parent data (10 correlation calculations total). This produces a damped sinusoidal function as expected [17]. It can be shown that the proper damping does result after a number of these correlations are performed. However, the application of additional correlations beyond that does numerically alter the extracted damping. Hence, this approach is not useful unless a metric is available to allow the analyst to know when to stop performing additional correlations. 

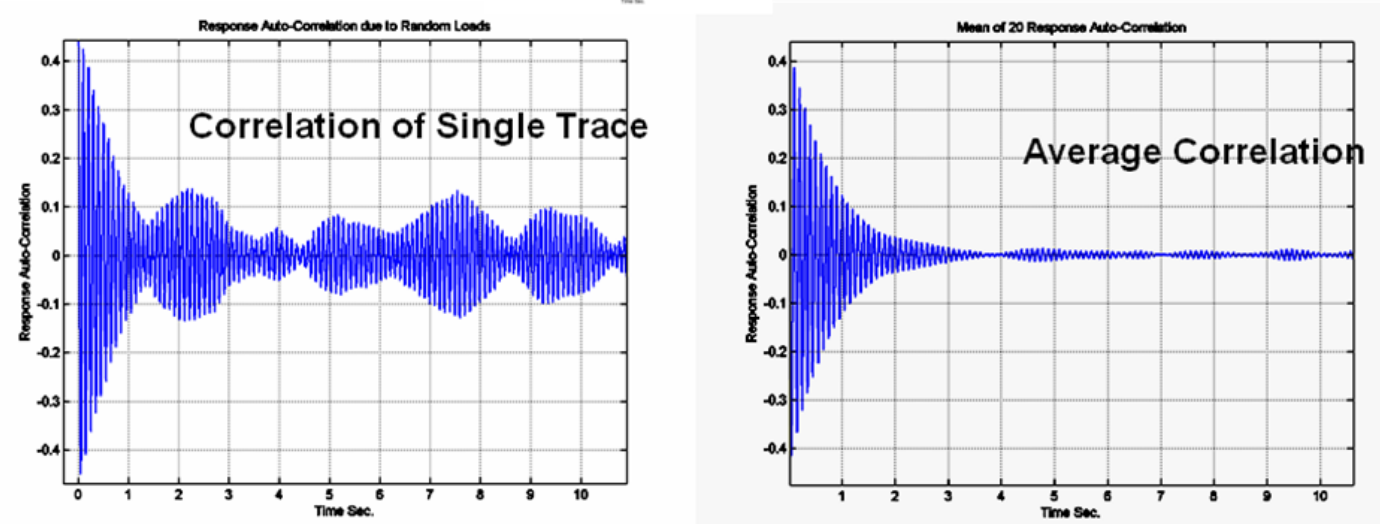

Figure 3. Use of Time Domain Averaging to Reduce Beating Phenomena in Correlation Functions
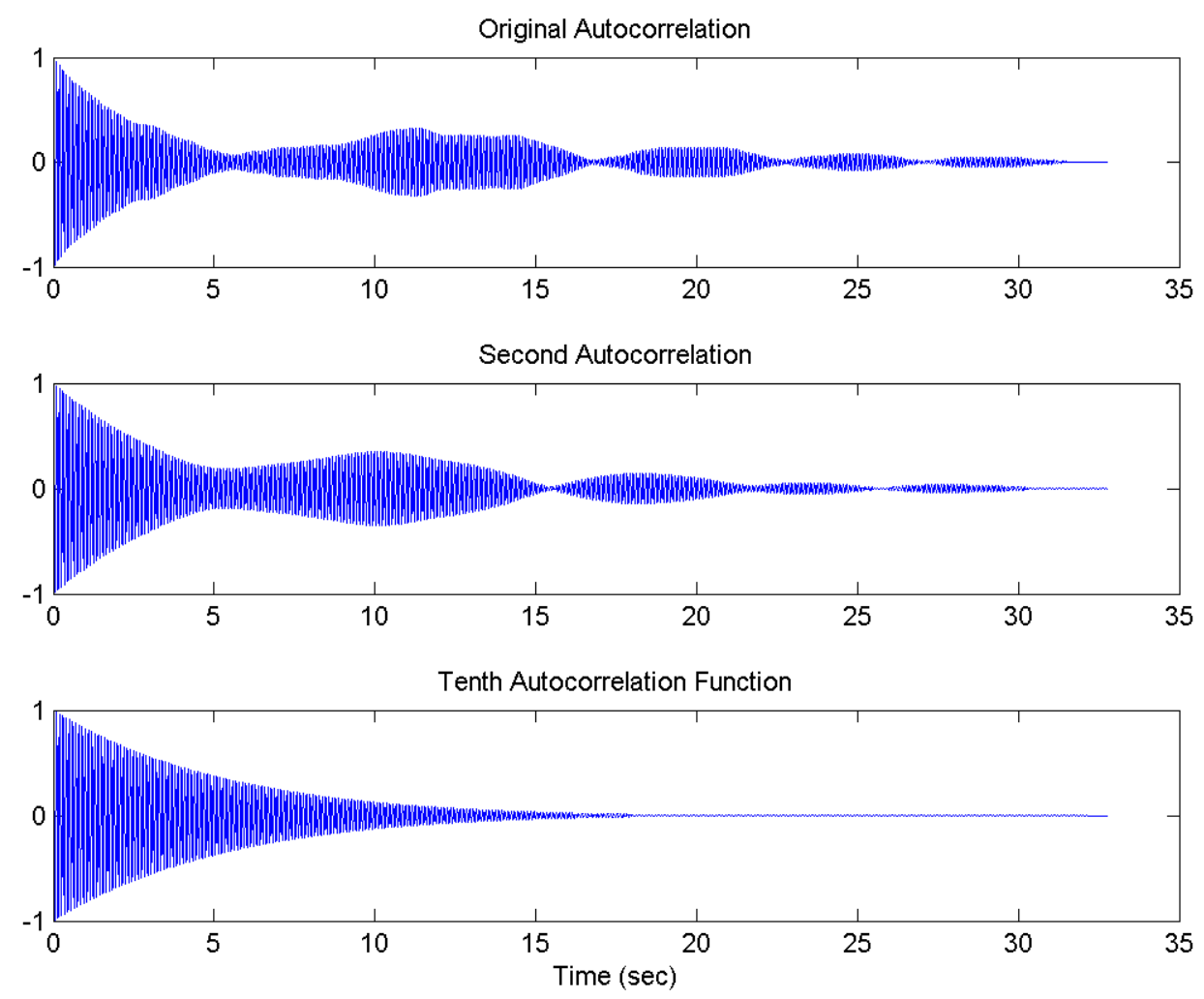

Figure 4. Use of Multiple Correlation Calculations to Reduce Beating Phenomena in Correlation Functions

The Maximum Entropy Method (MEM) was used in the 1980's to extend correlation functions using the earliest time lags to later time lags [11]. This approach is currently under assessment but is known to have significant sensitivity to the selected model order. This has proven to be the case with the most recent studies; however the assessments are not 
complete at this time. Another technique that has been used is similar situations with reasonable results is the Random Decrement technique $[13,14]$. This technique does produce correlation-type functions but the triggering process inherent in this approach may reduce the effects of the underlying modal excitations. Hence, this team is expecting to take a fresh look at this early OMA process.

In summary, there is a working hypothesis for the cause of the beating phenomena; which explains the inability of the previous solution approach (averaging correlation functions from multiple sensors [1]). Two approaches for solving the issue have been found (temporal averaging of correlations and multiple sequential correlations) but neither have general utility. A forth approach is under assessment (MEM) and a fifth is planned for assessment (Random Decrement).

\section{CONTROL SYSTEM EFFECTS}

Another question raised during the previous data processing studies was the interaction between the control system and the modal responses - primarily frequency and damping of the lowest bending modes [1]. An initial dataset has been acquired to begin studying the control system effects. This data set may also be used for some validation activities as well. The data is based on the Ares 1-X flight test. Ares 1-X was a full scale flight test vehicle for the Ares 1 vehicle that flew in 2009. The 327 foot tall vehicle consisted of a four segment Space Shuttle Solid Rocket Booster (SRB) configured as the first and only active stage. A mass simulator of a fifth segment, an upper stage, and the Orion vehicle were also present on the vehicle. Figure 5 shows the vehicle in-flight.

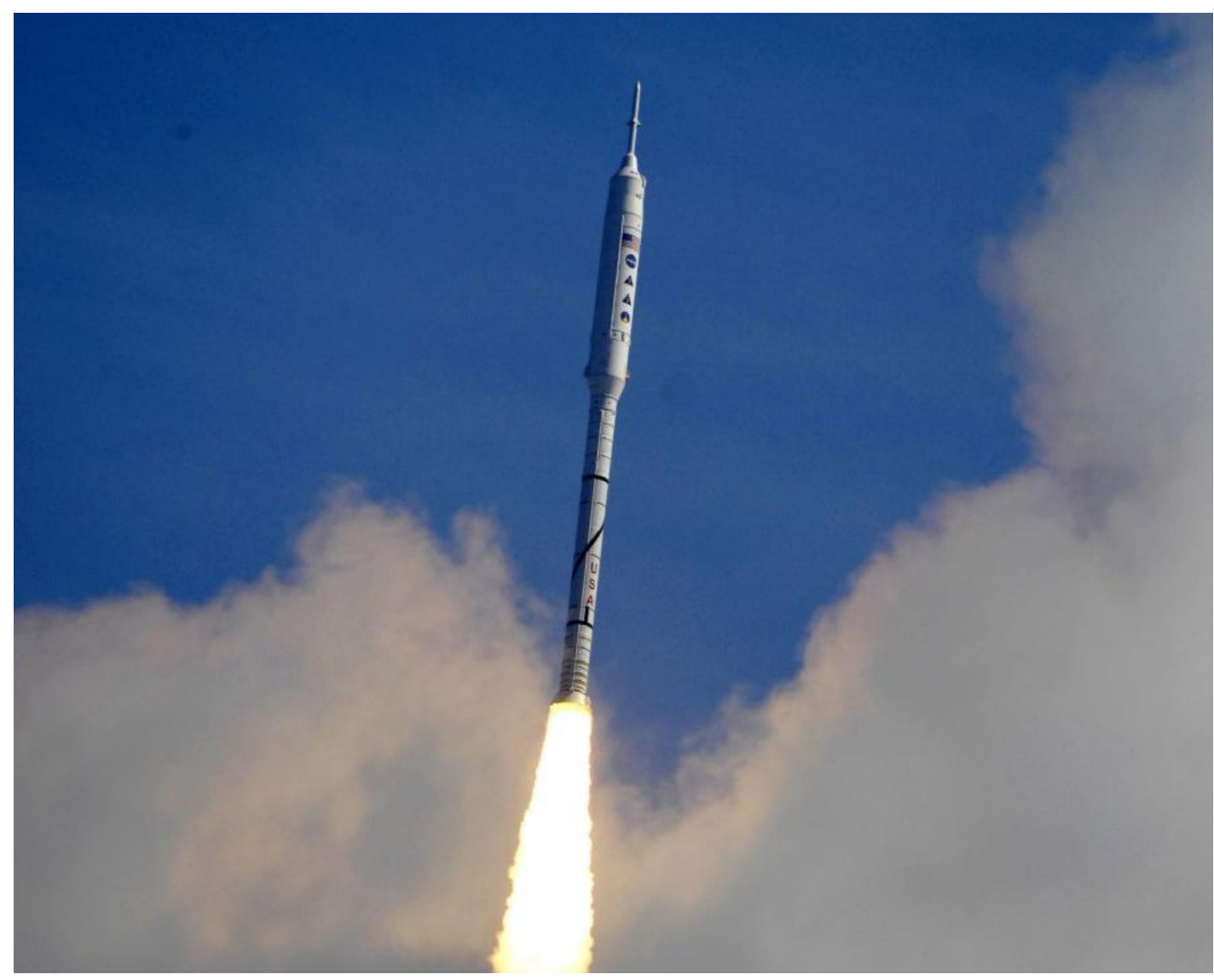

\section{Figure 5. Ares 1-X Vehicle in Flight}

Note that OMA work has been used by this team on Ares 1-X data during ascent and roll-out. Other teams have used OMA type approaches at other times during the build-up of the vehicle $[16,18]$. The latest dataset is a lift-off dataset that includes the first few seconds after pad release. Ten accelerometer measurements from the actual flight are provided 
with four measurements in the axial or flight direction and three measurements in each of the two tangential directions. Corresponding analytical predictions of the same measurement locations and times are also provided from a full-up analysis of the liftoff regime. These predictions have been developed with varying levels of complexity and completeness. Besides the full-up analysis with the wind loading and the flight controller effects, a thrust-only data set is provided as well as a dataset with the thrust and wind effects but no controller effects [19]. Although this dataset represents this team's first foray into understanding control system effects, the real control interactions of interest will not be seen until ascent as the pad release transients are a dominant loading. Additionally, since models of the system are available, studies related to hybrid force reconstruction techniques [8] may be performed.

\section{Axial Direction Data}

A natural breakdown of the data analysis (at least for the low frequency system modes) was to assess axial direction information separate from tangential direction information. An early step in the processing of the actual flight data was to determine if the modal properties are changing. A sliding window analysis of the four axial outputs was performed. For the analytical datasets, the time record was broken into 12 increments. Each increment was $26 \%$ of the total analysis time and each one was shifted by $6.5 \%$ of the total analysis time. The use of this unusual time metric of $\%$ of total analysis time is to avoid any export control data issues. The MATLAB "xcorr" function was used to generate auto-and cross correlation functions. Each increment was subjected to a $4^{\text {th }}$ order Butterworth digital filter applied with the MATLAB "filtfilt" command. The bandpass was from $1 \%$ of the sampling rate to $4 \%$ of the sampling rate. The pass band was chosen to contain the first two expected axial modes. As with the time data, the unusual frequency metric of \% of sampling frequency was chosen to avoid any export control issues. The Eigengensytem Realization Algorithm (ERA) was used to process one autocorrelation function and three cross-correlation functions from each increment assuming 20 modes. The complete 500 point correlation functions were used.

Figure 6 shows the sliding window analysis of the axial data from the analytical thrust+wind+controller dataset. The horizontal axis provides each of the 12 increments as a different "Case \#". The vertical axis provides the frequencies given as $\%$ of sampling frequencies. The color code provides the extracted damping ratio in $\%$ of critical. Note that the axial modes are expected at $2.2 \%$ and $3.3 \%$. The important information from this plot is that the modal frequencies do not significantly change over the total time analyzed. Hence, subsequent analyses of this dataset can be processed as if the data is stationary. Note that the other analytical data sets showed similar results.

Figure 7 provides the full time record (top plot), the linear frequency domain representation (middle plot), and semi-log frequency domain representation (bottom plot) of one of the axial sensors used for this analysis. Note that this dataset looks very much like a decaying sinusoid and converting to a correlation function is of limited utility. However it is a good exercise of some features of the processing software. The data was processed with the same filter and ERA parameters as the swept data discussed above. However, the entire dataset was used as one increment for this analysis. Time 0 was when the loads on the hold-down posts were as close to zero as possible. The extracted modes in this case were $2.2 \%$ with $.5 \%$ damping and $3.3 \%$ with $.7 \%$ damping. Figure 8 provides a synthesis of the autocorrelation function using these parameters. Note that the peak values and slopes of the two peaks were reproduced well. This suggests that the frequencies and damping estimations were reasonable for this dataset.

The actual flight data had to be processed slightly differently. The first and second axial modes were extracted separately. The first axial mode was estimated using sensor at the same four locations as the analytical data sets were using. The time records were again processed using approximately the same max time as the analytical data sets. The filter pass band went from $2 \%$ to $2.8 \%$ of the analytical sampling frequency. Since the flight data was sampled at a different rate than the analysis data was output, the analytical sample rate was used as the scale factor to allow a direct comparison between the two data sources. Figure 9 shows the filtered data set that was used for this analysis. Only $80 \%$ of the correlation function was used and the ERA run assumed two modes. The resulting mode was at $2.3 \%$ with .9\% damping. A synthesis of the $80 \%$ correlation function is shown in Figure 10. The modal frequency and damping appear to be captured appropriately. 


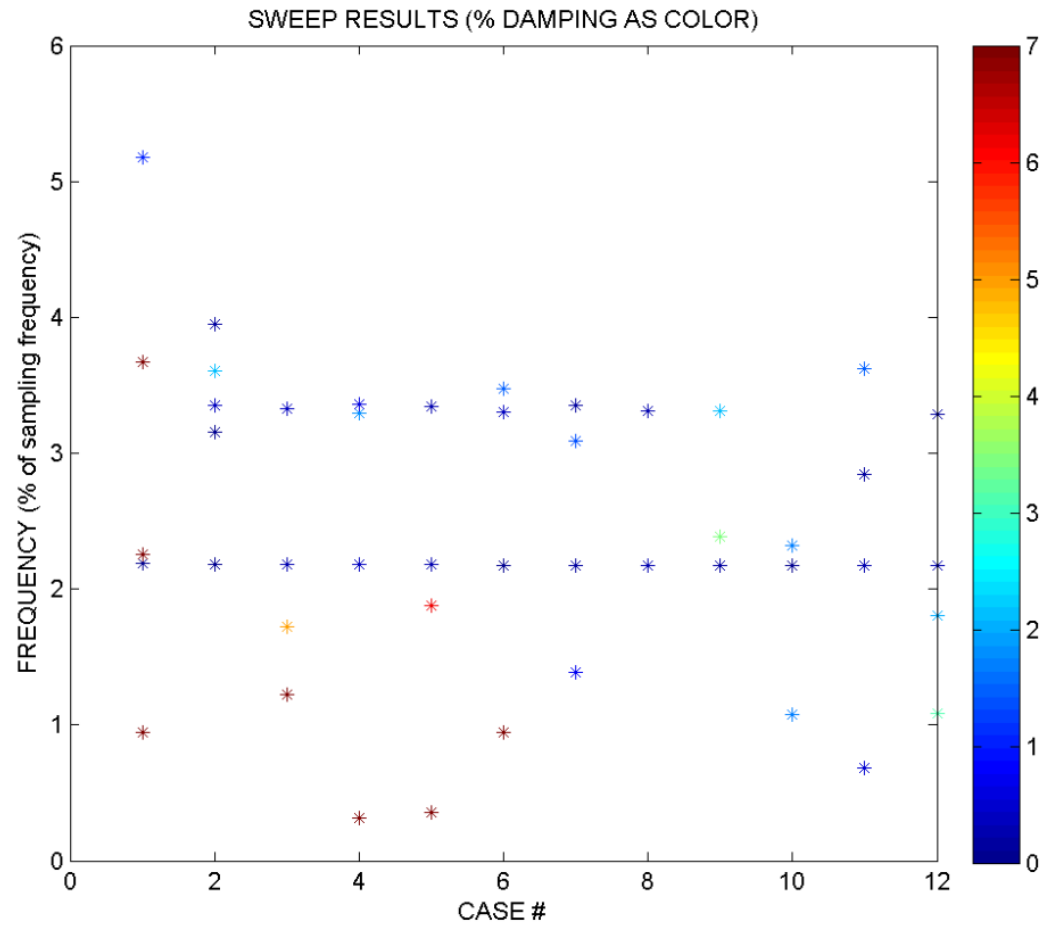

Figure 6. Sliding Window Analysis of the Ares 1-X Liftoff Thrust+Wind+Controller Data
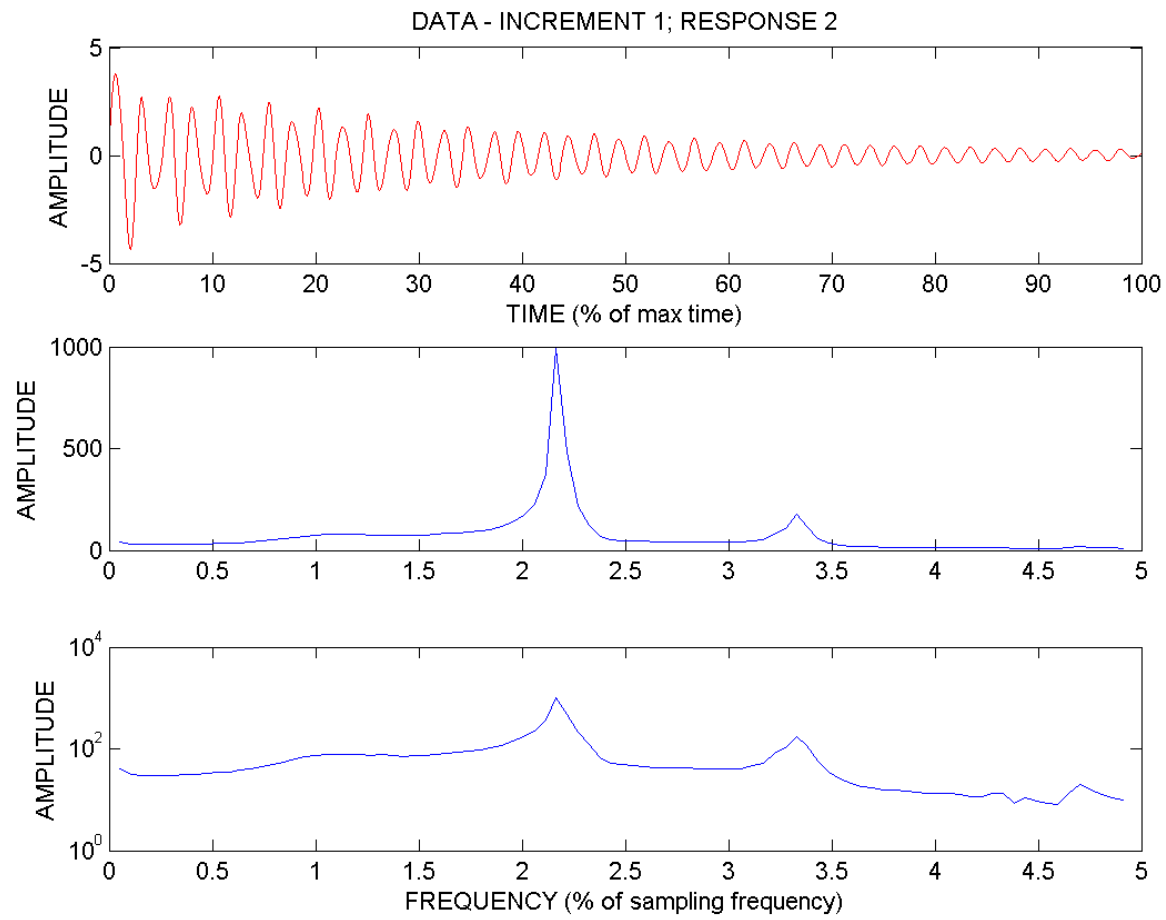

Figure 7. Time and Frequency Data of an Axial Output from the Ares 1-X Liftoff Thrust+Wind+Controller Set 

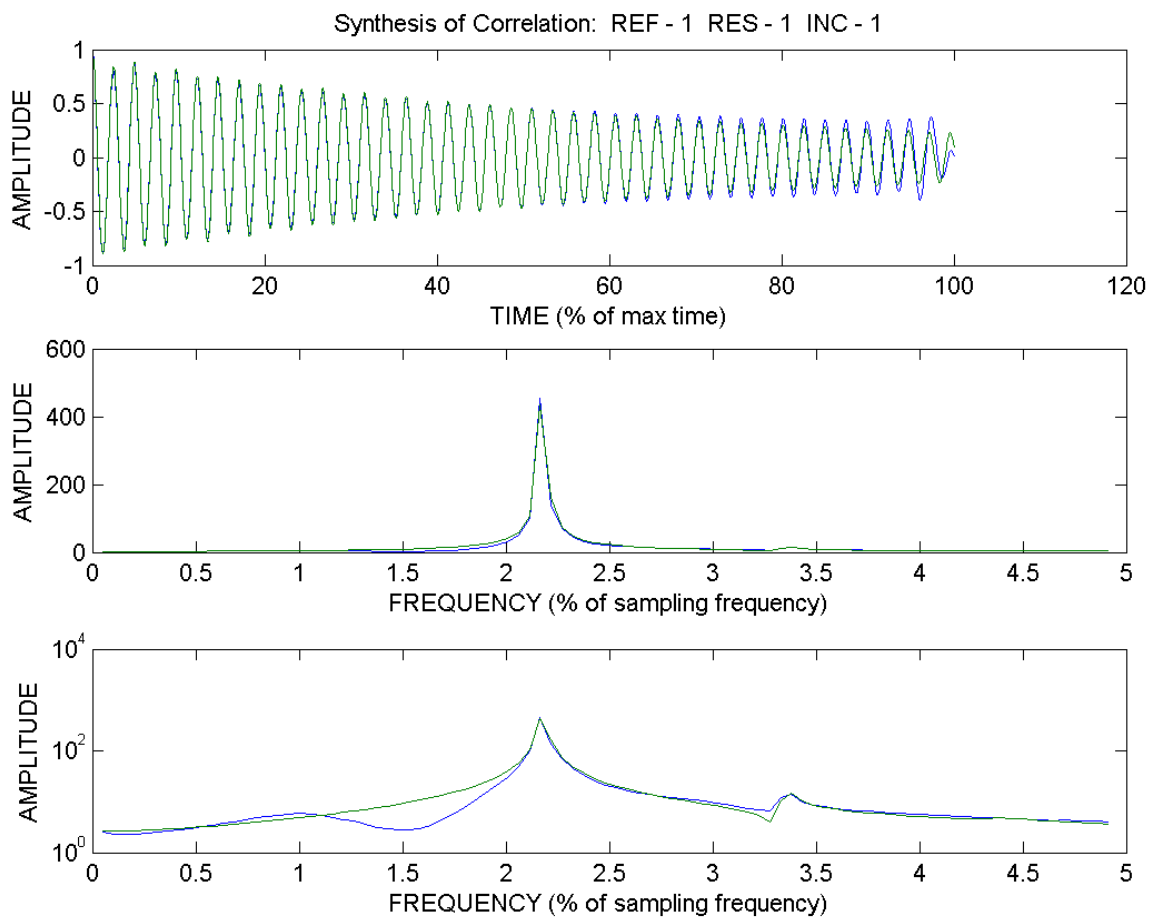

Figure 8. Axial Correlation Synthesis of an Output from the Ares 1-X Liftoff Thrust+Wind+Controller Set
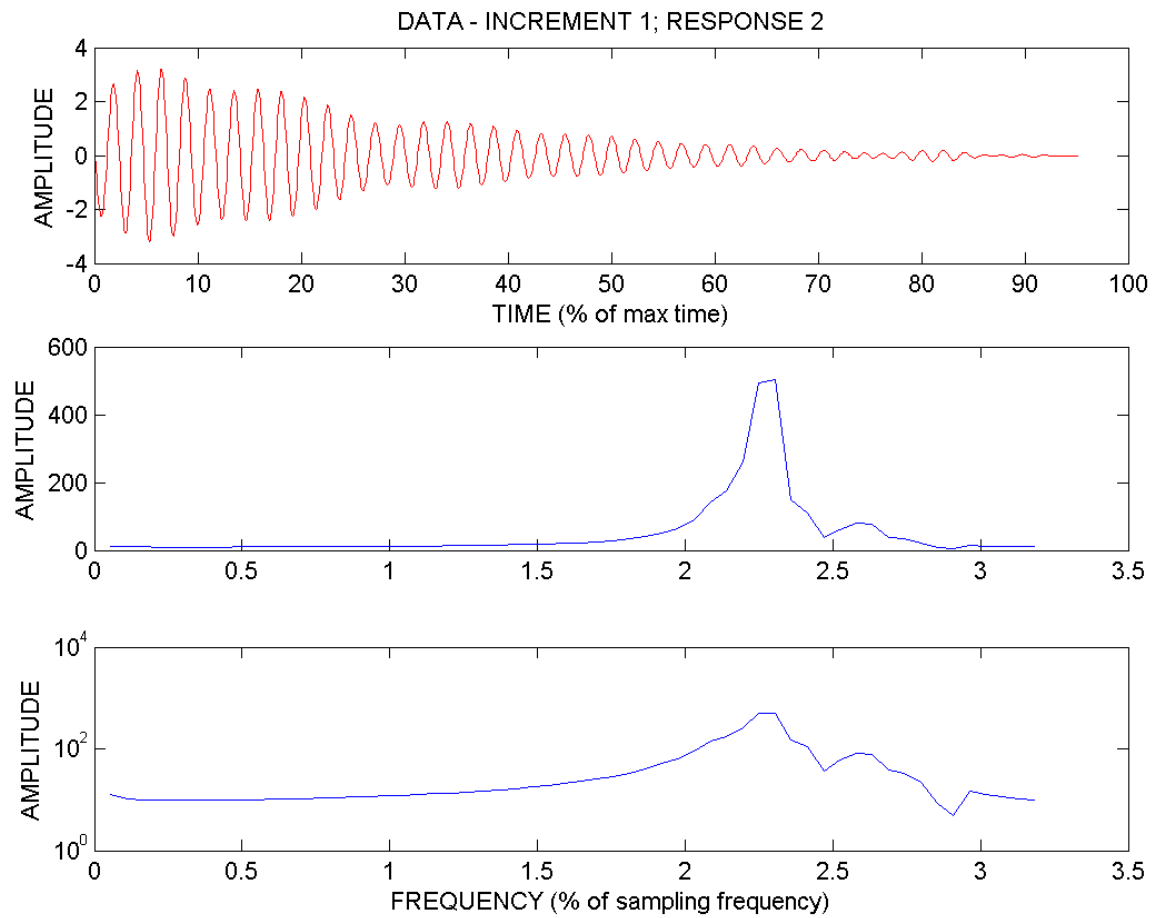

Figure 9. $1^{\text {st }}$ Axial Output of an Accelerometer from the Ares 1-X Liftoff Data 

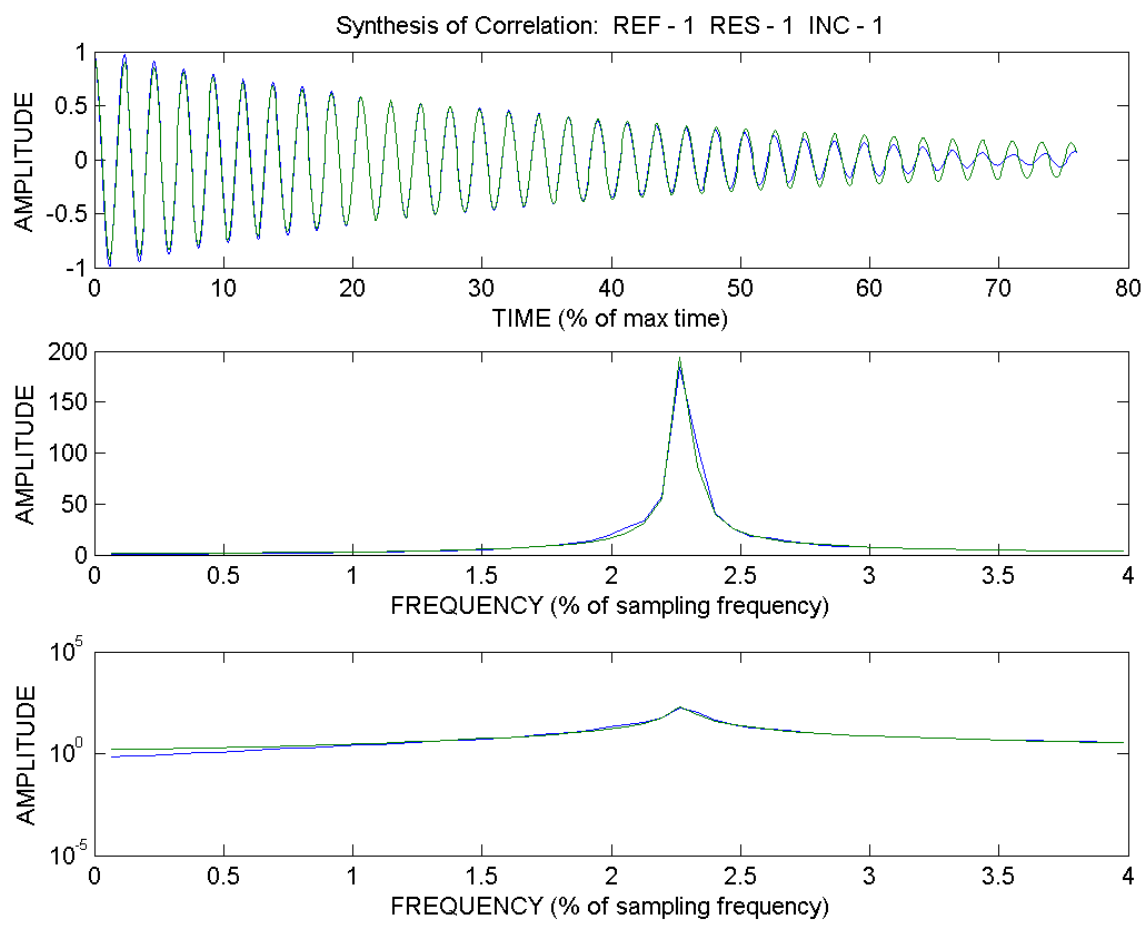

\section{Figure 10. $1^{\text {st }}$ Axial Correlation Synthesis of an Accelerometer from the Ares 1-X Liftoff Flight Data}

The second axial modal analysis was performed with only two of the axial sensors. The filter was set to $2.8 \%$ to $4 \%$ of the analytical sampling frequency. Six modes were expected by the ERA estimation. Figure 11 provides the synthesis of the autocorrelation of the same sensor output as provided in Figure 9. As expected, the situation is more complicated with real data than with the analysis as there appear to be two strong modes. Figure 12 provides the synthesis of the cross-correlation between the first and second axial sensors used in this analysis. The situation is again different as there only appears to be one strong mode. The modal estimation of this data set found viable modes at $2.9 \%$ with $1 \%$ damping; $3.4 \%$ with $1.5 \%$ damping; and $3.6 \%$ with $3.2 \%$ damping. More analysis would be required to fully sort out this second axial mode.

Table 1 provides a summary of the axial mode results. All three analytical data sets produced virtually the same modal frequencies and damping ratios. Furthermore, these frequencies were as expected from the known model inputs. There was no discernable impact of the control system on these results. However, the effect on the axial modes was not expected to be great. The flight data produced slightly higher frequency and damping values in the first mode. The second mode needs additional work to understand the results and make a reasonable comparison.

\section{Tangential Direction Data}

It was found that the tangential data analysis was more robust if the different modes were best processed individually. The $2^{\text {nd }}$ and $3^{\text {rd }}$ bending modes were extracted without significant difficulty but some additional improvements are possible. As with the axial modes, sliding window analyses suggested that the analytical datasets did not need to be analyzed as unsteady systems The Thrust+Wind+Controller dataset was analyzed for the $2^{\text {nd }}$ bending mode by using all six tangential outputs $4^{\text {th }}$ order Butterworth forward/reverse filtered outside of $.4 \%$ to $1 \%$ of the sampling rate. The MATLAB "xcorr" function was used to generate auto-and cross correlation functions. The Eigengensytem Realization Algorithm (ERA) was used to process one autocorrelation function and five cross-correlation functions assuming 20 modes. $80 \%$ of the 1893 data point time histories were used for processing. Figure 13 shows one filtered time history with one strong peak and other associated phenomena. Figure 14 shows the synthesis of the autocorrelation of the Figure 13 data. 

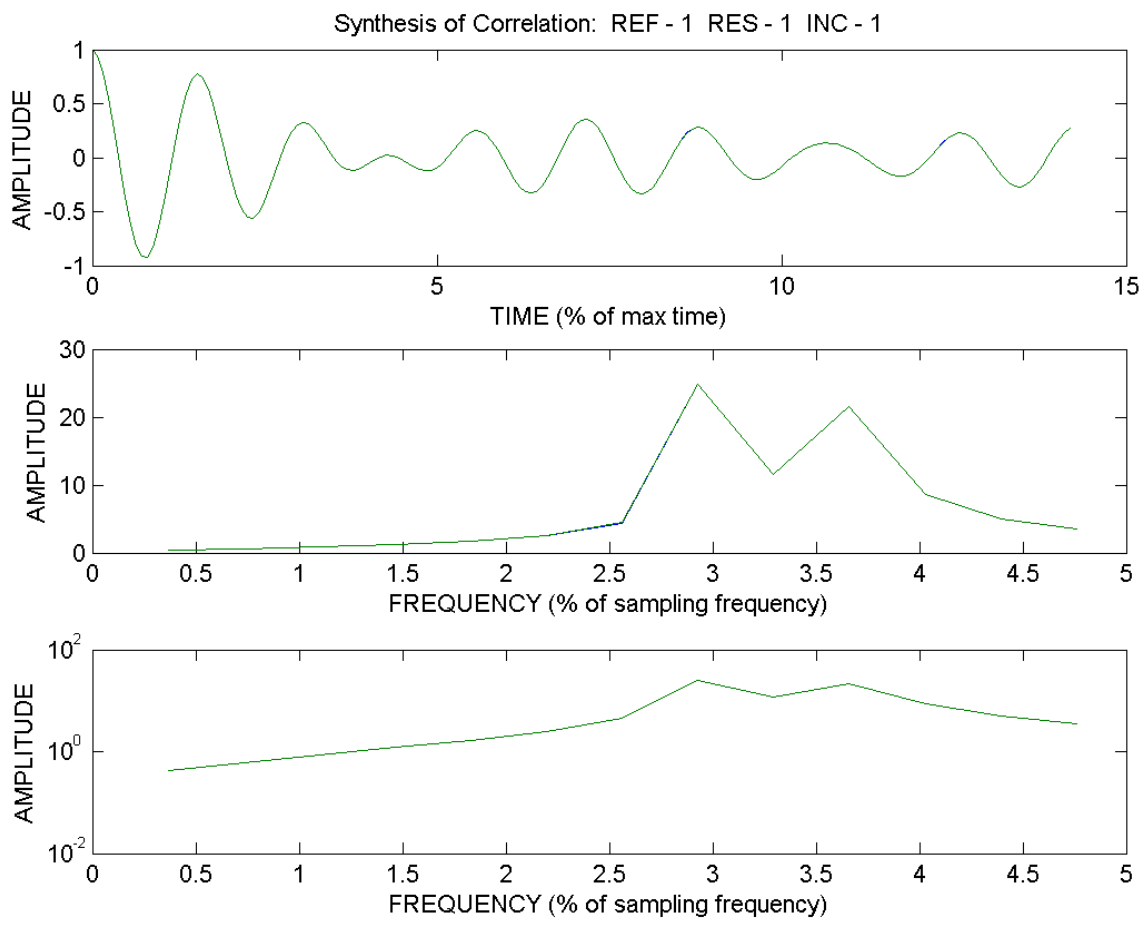

Figure 11. $2^{\text {nd }}$ Axial Output of an Accelerometer from the Ares 1-X Liftoff Data
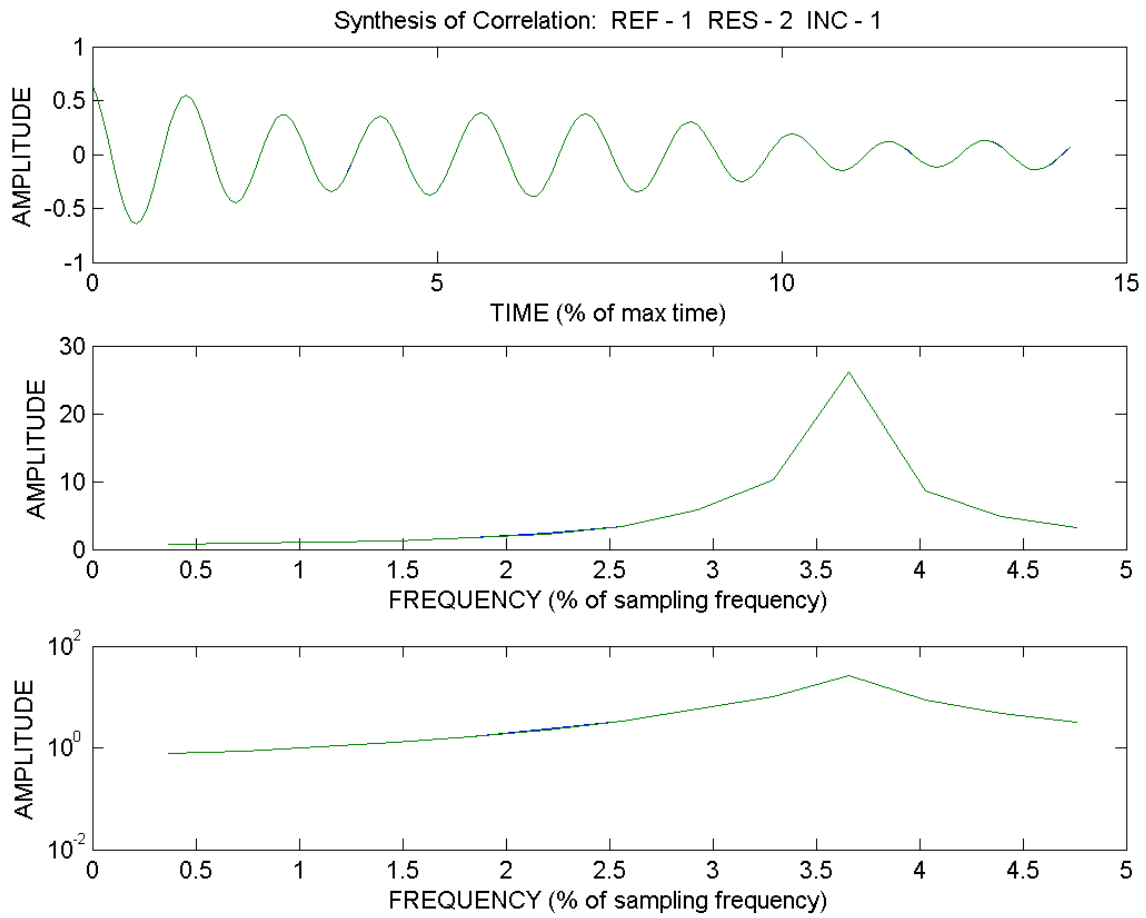

Figure 12. $2^{\text {nd }}$ Axial Correlation Synthesis of an Accelerometer from the Ares 1-X Liftoff Flight Data 
Table 1. $1^{\text {st }}$ and $2^{\text {nd }}$ Ares 1-X Axial Mode Results

\begin{tabular}{|l|c|c|c|c|}
\hline \multicolumn{2}{|c|}{$\begin{array}{c}1^{\text {st }} \text { Axial } \\
\text { Freq. (\%) }\end{array}$} & $\begin{array}{c}1^{\text {st }} \text { Axial } \\
\text { Damp (\%) }\end{array}$ & $\begin{array}{c}2^{\text {nd }} \text { Axial } \\
\text { Freq. (\%) }\end{array}$ & $\begin{array}{c}2^{\text {nd }} \text { Axial } \\
\text { Damp (\%) }\end{array}$ \\
\hline Thrust Only & 2.2 & 0.5 & 3.3 & 0.6 \\
\hline Thrust + Wind & 2.2 & 0.5 & 3.3 & 0.7 \\
\hline Thrust + Wind + Controller & 2.2 & 0.5 & 3.3 & 0.7 \\
\hline Flight & 2.3 & 0.9 & $2.9,3.4,3.6$ & $1.0,1.5,3.2$ \\
\hline
\end{tabular}
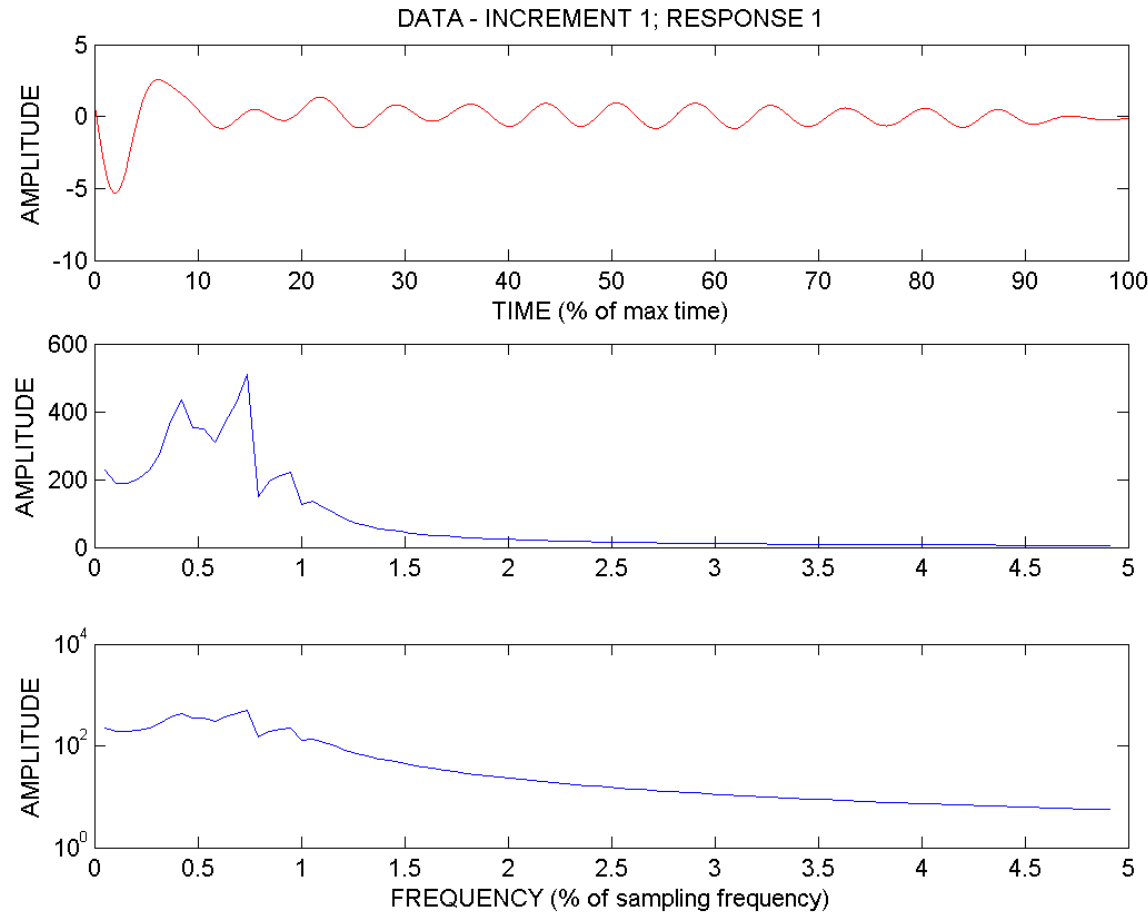

Figure 13. Tangential Output from the Ares 1-X Liftoff Thrust+Wind+Controller Set Targeting $2^{\text {nd }}$ Bending 

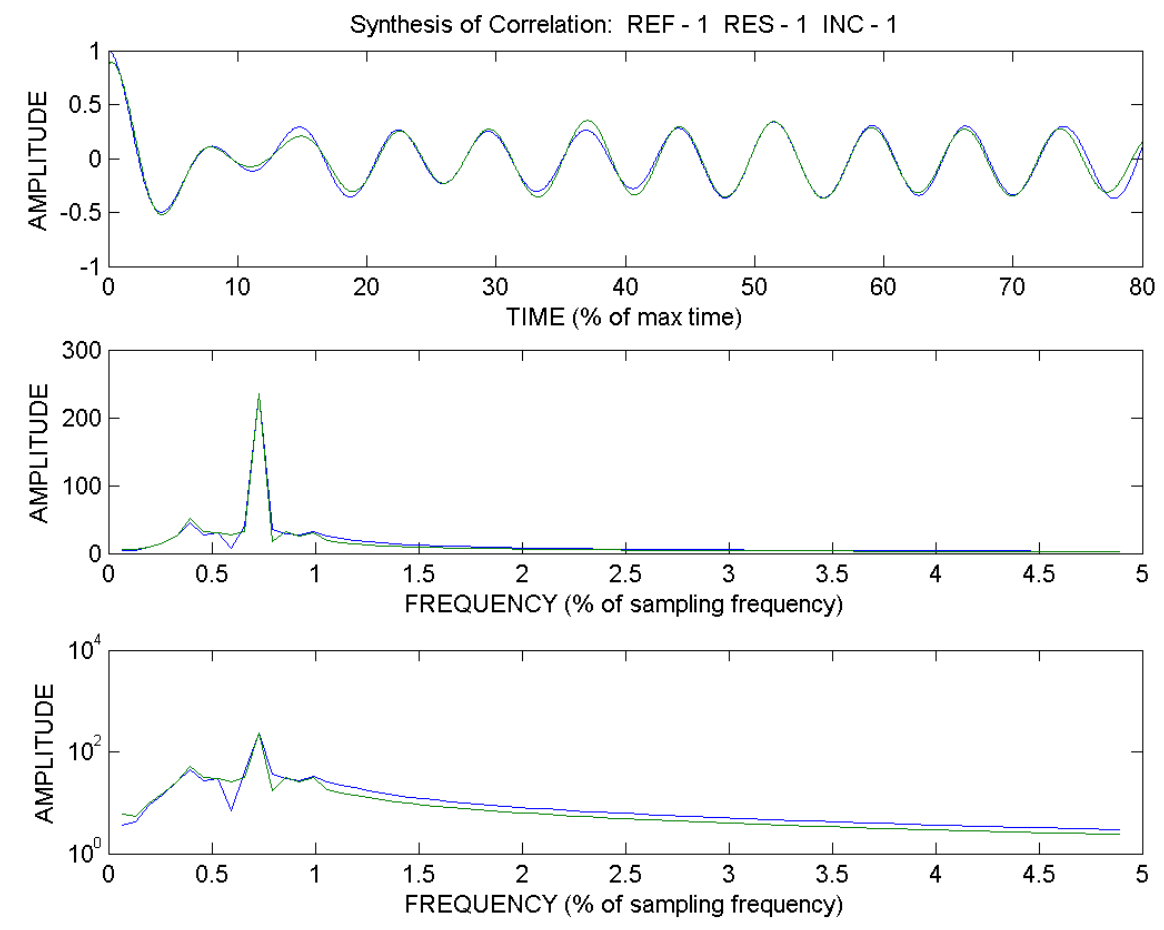

Figure 14. Tangential Correlation Synthesis of an Output from the Ares 1-X Liftoff Thrust+Wind+Controller Set

The other analytical data sets were processed with the same parameters as the Thrust + Wind + Controller for the $2^{\text {nd }}$ bending mode processing. The $2^{\text {nd }}$ bending flight data used $70 \%$ of the full correlation functions, which were also generated with the MATLAB "xcorr" function. The filter pass band for the flight data was $.4 \%$ to $1 \%$ of the analytical frequency. Five ERA modes were assumed. The pass band for the filter was $1 \%$ to $2 \%$ for the third bending mode analysis for the flight data as well as all three analytical data sets. All three analytical data sets and the flight data analyses used $80 \%$ of the available correlation functions from the same six sensors and processed with ERA assuming 5 modes. Table 2 provides the extracted frequency and damping results for the $2^{\text {nd }}$ and $3^{\text {rd }}$ bending modes from this exercise. There were some minor differences as the controller was added but there is not enough data here to make any generalizations.

Table 2. Ares 1-X $2^{\text {nd }}$ and $3^{\text {rd }}$ Bending Mode Results

\begin{tabular}{|c|c|c|c|c|}
\hline & $\begin{array}{l}2^{\text {nd }} \text { Bending } \\
\text { Freq. (\%) }\end{array}$ & $\begin{array}{c}2^{\text {nd }} \text { Bending } \\
\text { Damp (\%) }\end{array}$ & $\begin{array}{l}3^{\text {rd }} \text { Bending } \\
\text { Freq. (\%) }\end{array}$ & $\begin{array}{l}3^{\text {rd }} \text { Bending } \\
\text { Damp (\%) }\end{array}$ \\
\hline Thrust Only & .74 & 0.1 & 1.4 & 0.4 \\
\hline Thrust + Wind & .74 & 0.1 & 1.4 & 0.4 \\
\hline Thrust + Wind + Controller & .72 & 0.3 & 1.4 & 0.5 \\
\hline Flight & .78 & 0.6 & 1.5 & 0.5 \\
\hline
\end{tabular}


The first bending mode has been a difficult mode to extract from ascent flight data [16]. This liftoff data and analytical has also proven to be a difficult data set from which to extract the first bending mode. Figure 15 shows one of the six outputs used to estimate the modal parameters after filtering from $0 \%$ to $.4 \%$. The analytical data was found to provide $1^{\text {st }}$ bending modes most readily after decimating the data by 10 times. Note that the MATLAB function "decimate" was used to filter and then decimate. The Figure 15 data was decimated before plotting. The synthesized correlation function is provided in Figure 16. It is fairly obvious from Figures 15 and 16 that longer correlation functions are needed to allow this low frequency mode fully express itself in the correlation data.
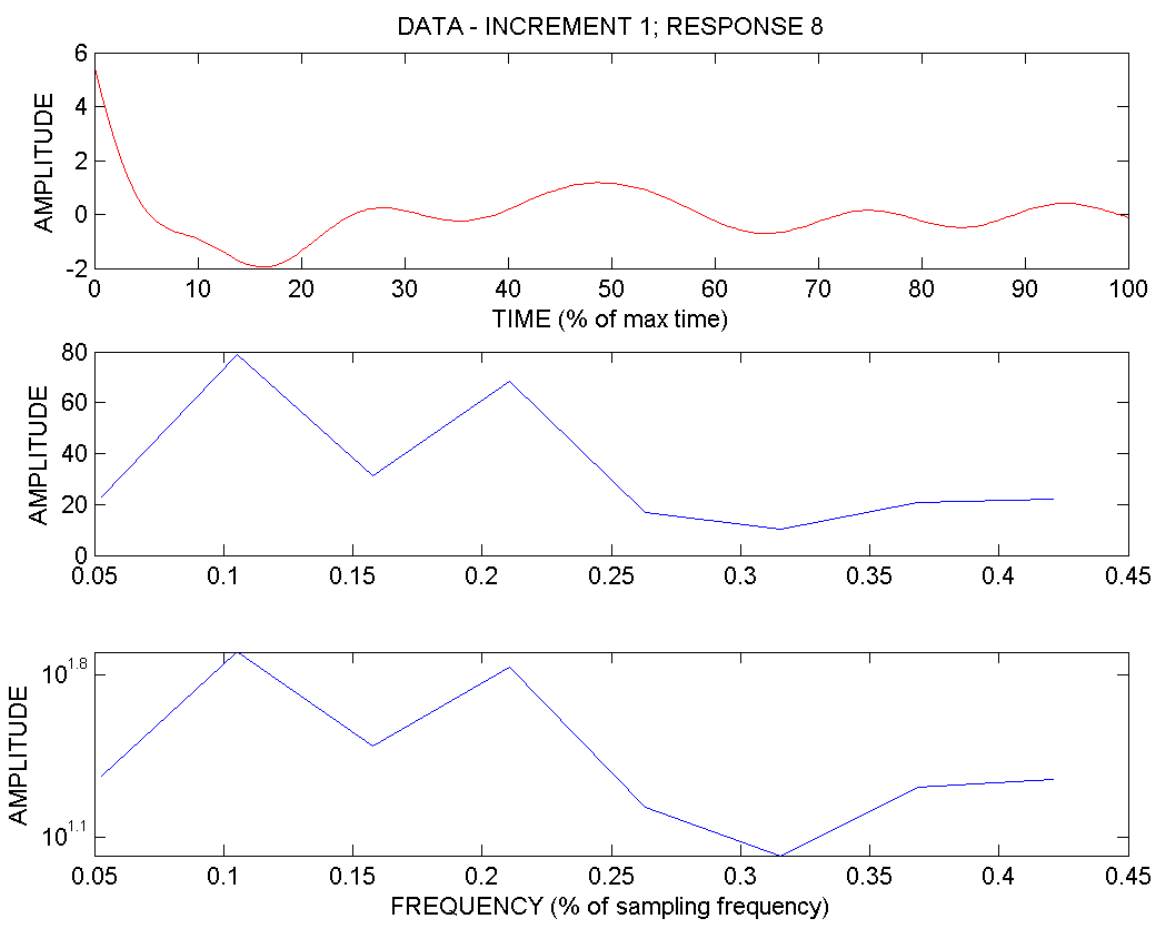

\section{Figure 15. Tangential Output from the Ares 1-X Liftoff Thrust+Wind+Controller Set Targeting $1^{\text {st }}$ Bending}

Table 3 contains the results of the first bending mode for the vehicle. It is obvious here as well that the consistency of current approaches have broken down with this data set for the first bending mode. The intent was to process all three analytical data sets with the same parameters. In this case those data sets were processed with all eight tangential sensors after decimating by 10 and filtering from $0 \%$ to $.4 \%$ of the undecimated original analytical data rate. ERA was expecting 10 modes for all three analyses. $80 \%$ of the six correlation functions were used for processing. The flight data processed all six tangential sensors as well but used a different reference channel for correlation calculations. $70 \%$ of the correlations functions were used for analysis. The filter band pass was between $.1 \%$ and $.6 \%$ of the analytical sampling rate. The ERA identification was expecting 5 modes. The flight data was not decimated. It appears that the decimation was critical to getting the data into a proper range for the identification. It may be that decimation would have helped the identification of the $2^{\text {nd }}$ bending mode as well.

\section{Control System Observations}

There are indications that the control system can systematically affect damping as expected. However, this data set alone is not appropriate to drive general statements. Longer data sets including those at later more challenging flight times would be needed. This data set is excellent to help begin the validation process as the models are available and the data had been provided in increasing steps of complexity. Additional work with this data set will be performed in the future. 

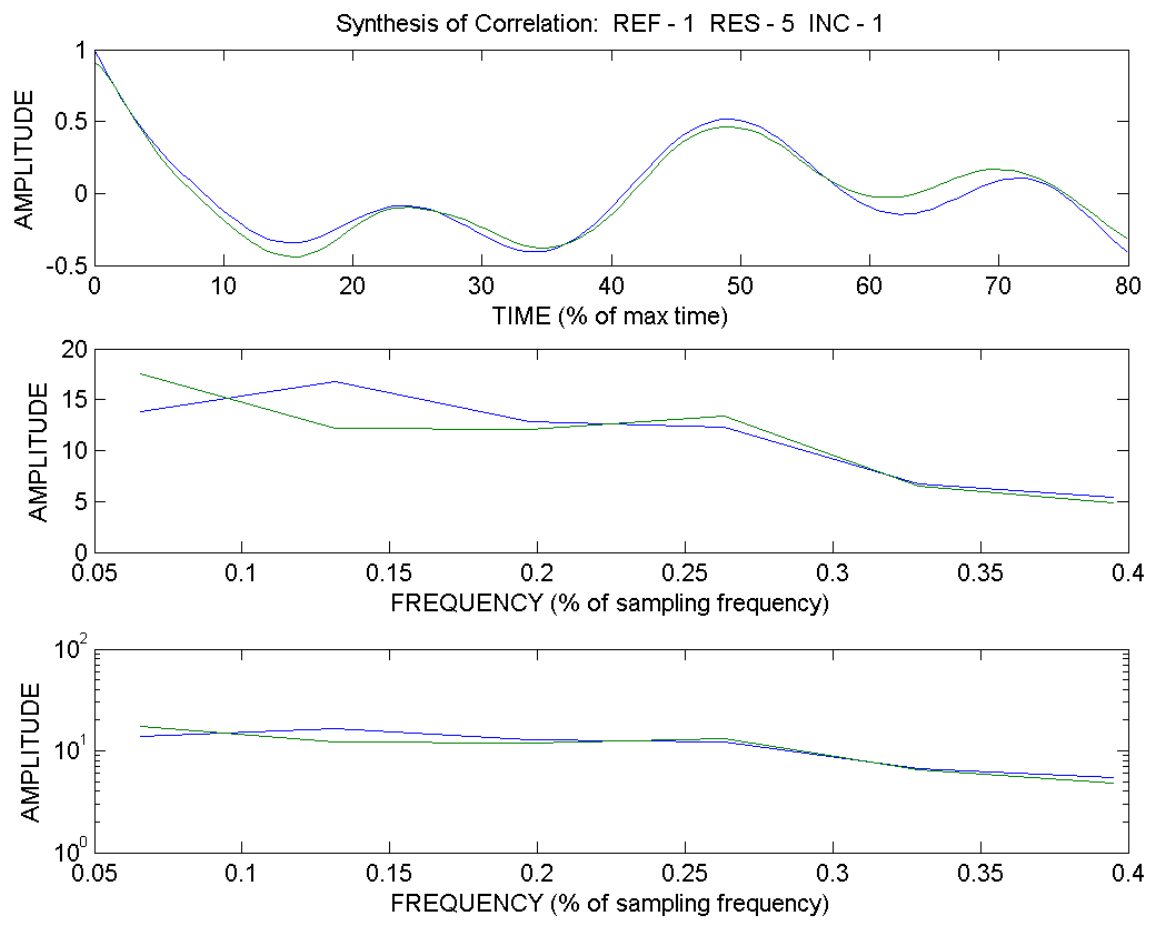

Figure 16. Correlation Synthesis the Ares 1-X Liftoff Thrust + Wind+Controller Set Targeting $1^{\text {st }}$ Bending

Table 3. Ares 1-X $1^{\text {st }}$ Bending Mode Results

\begin{tabular}{|l|c|c|}
\hline \multicolumn{2}{|c|}{$\begin{array}{c}1^{\text {st }} \text { Bending } \\
\text { Freq. (\%) }\end{array}$} & $\begin{array}{c}1^{\text {st }} \text { Bending } \\
\text { Damp (\%) }\end{array}$ \\
\hline Thrust Only & .28 & 0.4 \\
\hline Thrust + Wind & .26 & 2.7 \\
\hline Thrust + Wind + Controller & .22 & 1.9 \\
\hline Flight & .30 & 3.6 \\
\hline
\end{tabular}

\section{SUMMARY AND CONCLUSIONS}

The difficult liftoff and ascent launch vehicle environment has shown to be amenable to in-situ processing in the past. Therefore, the goal of obtaining a common set of tools to perform operational modal analysis on a wide range of flight vehicles and program milestones is still viable. However, it has been found that the launch environment does have some vexing issues that still need to be worked. One of these issues is the beating phenomena seen in the short time records. The current hypothesis is that this phenomenon is due to numerical increases in correlation due to the periodic but ran- 
dom excitations of the modal system. These phenomena can be reduced by averaging in the time or frequency domain or by taking multiple correlation processing steps. The Maximum Entropy Method and the Random Decrement methods are being assessed to also assist in overcoming the beating phenomena.

The next issue is the potential adjustment of flight loads by the vehicle control system during flight. An early Ares 1 -X data base has been used to begin the studies of controller effect. However more extensive later time records are needed fully address this issue. However, there are some interesting consistencies and simplifications in this data set that make the Ares 1-X liftoff data a good candidate for using in later validation exercises.

Development work is expected to meet the needs for the full-up launch analysis tools including the activities mentioned above. Additional ongoing activities include continuing development of the MIDOS code, module development for additional OMA algorithms, operational analyses of existing and new datasets (including analytical data), and validation activities for the final implementation. On a parallel track, development activities are also needed to extend the OMA technologies into the needs for high-frequency analyses. All of these activities are to address the need to use operational modal analysis to provide for diagnostic testing in multiple niches during the life of a spacecraft vehicle development.

\section{REFERENCES}

1. James G, Cao T, Fogt V, Wilson R L, and Bartkowitz, T (2011) Extraction of Modal Parameters from Spacecraft Flight Data. Proceedings of the $29^{\text {th }}$ International Modal Analysis Conference.

2. James G, Carne T, and Marek E (1994) In-situ modal analysis of STARS missile flight data and comparison to pre-flight predictions from test-reconciled models. Proceedings of the $15^{\text {th }}$ Institute of Environmental Sciences Aerospace Testing Seminar.

3. Goursat M, Döhler M, Mevel L, and Andersen P (2010) Crystal Clear SSI for Operational Modal Analysis of Aerospace Vehicles. Proceedings of the $28^{\text {th }}$ International Modal Analysis Conference.

4. Zhang L, Wang T, and Tamura Y (2010) A Frequency-Spatial Domain Decomposition (FSDD) method for operational modal analysis. Mechanical Systems and Signal Processing, Vol. 24, No. 5, pp. 1227-1239.

5. Freudinger L, Lind R, and Brenner M (1997) Correlation Filtering of Modal Dynamics using the Laplace Wavelet. NASA CR-97207066.

6. Huang, et al. (1998) The empirical mode decomposition and the Hilbert spectrum for non-linear and non-stationary time series analysis. Proccedings of the Royal Society of London, 454, 903-993.

7. Bjerg I, Hansen S, Brinker R, and Aenlle M L (2007) Load estimation by frequency domain decomposition. Proceedings of the $2^{\text {nd }}$ International Operational Modal Analysis Conference, pp 669-676.

8. James G, Carne T, and Wilson B (2007) Reconstruction of the Space Shuttle roll-out forcing function. Proceedings of the $25^{\text {th }}$ International Modal Analysis Conference.

9. Hermans L and Van der Auweraer H (1997) On the use of auto and cross-correlation functions to extract modal parameters from output-only data. Proceedings of Structural Dynamics: Recent Advances, Southhampton, U.K., July 14-17.

10. Mohanty P and Rixen D (2003) Modifying the ERA identification for operational modal analysis in the presence of harmonic perturbations. Proceedings of the $16^{\text {th }}$ ASCE Engineering Mechanics Conference, Seattle, July 16-18.

11. Campbell R and Vandiver J (1980) The estimation of natural frequencies and damping ratios of offshore structures. Proceedings of the $12^{\text {th }}$ Annual Offshore Technology Conference.

12. Briggs M (1981) Multichannel maximum entropy method of spectral analysis applied to offshore structures. WHOI-81-69, Woods Hole Oceanographic Institute.

13. Ibrahim, S (1977) Random decrement technique for modal identification of structures. Journal of Spacecraft and Rockets, Vol. 14, N. 4, pp. 183-198.

14. Asmussen J, Ibrahim S, and Brinker R (1998), Random Decrement: Identification of Structures Subjected to Ambient Excitation. Proceedings of the $16^{\text {th }}$ International Modal Analysis Conference.

15. James G (2003) Modal parameter estimation from Space Shuttle flight data. Proceedings of the $21^{\text {st }}$ International Modal Analysis Conference.

16. Bartkowicz T and James G (2011) Ares 1-X in-flight modal identification. Proceedings of the American Institute of Aeronautics and Astronautics Structures, Structural Dynamics, and Materials Conference.

17. James G, Carne T, and Lauffer J (1995) The Natural Excitation Technique (NExT) for modal parameter extraction from operating structures. SEM International Journal of Analytical and Experimental Modal Analysis, Vol. 10, No. 4.

18. Templeton J, Buerhrle R, Gaspar J, Parks R, and Lazor D (2010) Ares 1-X Launch Vehicle Modal Test Measurements and Data Quality Assessments. Proceedings of the XXVIII International Modal Analysis Conference.

19. Alldredge D (2011) OMA Data Delivery. DCI-TM-081011-1050-01, Dynamic Concepts, Inc., Huntsville, AL. 